\title{
Cystic Fibrosis Transmembrane Regulator Correction Attenuates Heart Failure-Induced Lung Inflammation
}

\author{
Franziska Uhl ${ }^{1}$, Lotte Vanherle $^{1}$, and Anja Meissner ${ }^{1}$ \\ ${ }^{1}$ Lunds Universitet
}

October 16, 2021

\begin{abstract}
Background and Purpose: Heart failure (HF) affects 64 million people worldwide. Despite advancements in prevention and therapy, quality of life remains poor for many HF patients due to target organ damage. Pulmonary manifestations of HF are well-established. However, difficulties in the treatment of HF patients with chronic lung phenotypes remain, as standard therapies are often complicated by contraindications. Here, we verify the downregulation of the cystic fibrosis transmembrane regulator (CFTR) in the HF lung, a concept that may provide new mechanism-based therapies for HF patients with pulmonary complications. Experimental Approach: Ligation of the left anterior descending coronary artery in mice was used to induce myocardial infarction (MI). At 10 weeks post-MI, pharmacological CFTR corrector therapy (Lumacaftor (Lum)) was applied systemically or lung-specific for 2 weeks, and the lungs were analysed using histology, flow cytometry, Western blotting, and qPCR. Key Results: Experimental HF associated with an apparent lung phenotype characterized by reduction of pulmonary CFTR + cells, vascular remodelling, and pronounced tissue inflammation as evidenced by infiltration of pro-inflammatory monocytes and elevation of classically-activated macrophages in the lung. PharmacologicalCFTR correction with Lum mitigated the HF-induced downregulation of pulmonary CFTR expression, increased the proportion of CFTR + cells in the lung, and diminished the HF-associated elevation of classically-activated non-alveolar macrophages within the lungs with implication for vessel wall thickness. Conclusion and Implications: Collectively, our data suggest that pharmacological CFTR correction possesses the capacity to alleviate HF-induced inflammation in the lung and may emerge as treatment option for HF patients with chronic lung phenotypes.
\end{abstract}

Cystic Fibrosis Transmembrane Regulator Correction Attenuates Heart Failure-Induced Lung Inflammation

Short running title: CFTR and lung inflammation during HF

Franziska E. Uhl ${ }^{1,2}$, franziuhl82@gmail.com, ORCID: 0000-0002-0641-4162

Lotte Vanherle ${ }^{1,2}$ lotte.vanherle.8386@med.lu.se, ORCID: 0000-0002-4864-1347

Anja Meissner ${ }^{1,2}$ anja.meissner@med.lu.se, ORCID: 0000-0002-0793-7213

${ }^{1}$ Department of Experimental Medical Science, Lund University, Lund, Sweden.

${ }^{2}$ Wallenberg Centre for Molecular Medicine, Lund University, Lund, Sweden.

Correspondence to: Anja Meissner; Klinikgatan 32, SE-22184 Lund, Sweden; anja.meissner@med.lu.se; $+46(0) 462220641$

Data availability: Data underlying this article are available in the article and in its online supplementary material or are available from the corresponding author upon reasonable request. 
Funding statement: This work was supported by the following funding sources: Knut and Alice Wallenberg foundation [AM], Swedish Research Council [VR; 2017-01243; AM]; German Research Foundation [DFG; ME 4667/2-1; AM]; Åke Wibergs Stiftelse [M19-0380; AM]; Albert Påhlssons Stiftelse [AM], Inger Bendix Stiftelse [AM-2019-10; AM], Stohnes Stiftelse [AM]; Crafoord Foundation [FU], Royal Physiographic Society Lund [FU], STINT [MG19-8469; AM], and Lund University [AM].

Author contribution statement: Conceptualization, AM; methodology, FEU and AM; validation, FEU and AM; formal analysis, FEU, LV; resources, AM; data curation, FEU, LV; writing - original draft preparation, FEU, AM; writing - review and editing, FEU, LV and AM.; visualization, FEU and AM; supervision, AM; project administration, AM; funding acquisition, FEU and AM. All authors have read and agreed to the published version of the manuscript.

Conflict of interest disclosure: The authors declare no conflict of interest.

Ethics approval statement: This investigation conforms to the Guide for Care and Use of Laboratory Animals published by the European Union (Directive 2010/63/EU) and with the ARRIVE guidelines. All animal care and experimental protocols were approved by the institutional animal ethics committee at Lund University (Dnr.: 5.8.18-08003/2017, 5.8.18-04938/2021) and were conducted in accordance with European animal protection laws.

Permission to reproduce material from other sources: Only original material was used.

Manuscript category: Research article

\title{
Word Count: 3057
}

\section{Acknowledgements}

The authors thank the Knut and Alice Wallenberg foundation for generous support and the Lund University BioImaging Center (LBIC), Lund University is gratefully acknowledged for providing experimental resources. We further like to thank René In 't Zandt and Michael Gottschalk from LBIC for help with the cardiac MRI analyses, Dr. Steffen-Sebastian Bolz and Dr. Darcy Lidington (both Department of Physiology, University of Toronto, Toronto, Canada) for providing lung tissue, Dr. Nicholas Don-Doncow (Department of Experimental Medical Science, Lund University, Lund, Sweden) for discussing FACS panel design, Dr. Gunilla WestergrenThorsson (Department of Experimental Medical Science, Lund University, Lund, Sweden) for access to the microtome, Dr. Jonas Erjefält (Department of Experimental Medical Science, Lund University Sweden) for access to the paraffin embedding machine, Dr. Darcy Wagner (Department of Experimental Medical Science, Lund University, Lund Sweden) for access to the tissue processor, Dr. Ulrica Englund Johansson (Department of Clinical Sciences Section IV, Lund University, Lund Sweden) for access to the Zeiss Axio Imager, and Dr. Björn Olde (Department of Clinical Sciences Section II, Lund University Sweden) for the RAW246.7 cells.

\begin{abstract}
Background and Purpose: Heart failure (HF) affects 64 million people worldwide. Despite advancements in prevention and therapy, quality of life remains poor for many HF patients due to target organ damage. Pulmonary manifestations of HF are well-established. However, difficulties in the treatment of HF patients with chronic lung phenotypes remain, as standard therapies are often complicated by contraindications. Here, we verify the downregulation of the cystic fibrosis transmembrane regulator (CFTR) in the HF lung, a concept that may provide new mechanism-based therapies for HF patients with pulmonary complications.
\end{abstract}

Experimental Approach: Ligation of the left anterior descending coronary artery in mice was used to induce myocardial infarction (MI). At 10 weeks post-MI, pharmacological CFTR corrector therapy (i.e., Lumacaftor (Lum)) was applied systemically or lung-specific for 2 weeks, and the lungs were analysed using histology, flow cytometry, Western blotting, and qPCR.

Key Results: Experimental HF associated with an apparent lung phenotype characterized by reduction of pulmonary $\mathrm{CFTR}^{+}$cells, vascular remodelling, and pronounced tissue inflammation as evidenced by 
infiltration of pro-inflammatory monocytes and elevation of classically-activated macrophages in the lung. Pharmacological correction of CFTR with Lum mitigated the HF-induced downregulation of pulmonary CFTR expression, increased the proportion of $\mathrm{CFTR}^{+}$cells in the lung, and diminished the HF-associated elevation of classically-activated non-alveolar macrophages within the lungs with implication for vessel wall thickness.

Conclusion and Implications: Collectively, our data suggest that pharmacological CFTR correction possesses the capacity to alleviate HF-induced inflammation in the lung and may emerge as treatment option for HF patients with chronic lung phenotypes.

\section{Keywords}

Lung, heart failure, cystic fibrosis transmembrane regulator, inflammation

\section{What is already known:}

Pulmonary manifestations of heart failure (HF) are well-established.

HF reduces pulmonary cystic fibrosis transmembrane regulator (CFTR) expression

\section{What this study adds:}

CFTR as molecular link between lung complications and HF.

Lung inflammation accompanying HF can be attenuated by pharmacological correction of pulmonary CFTR expression.

\section{Clinical significance:}

Clinically approved CFTR corrector can reduce pulmonary complications during HF.

Opens the door for available CF therapeutics with long-term safety profiles to managing pulmonary complications during HF.

\section{Introduction}

Heart failure (HF) currently affects 64 million people worldwide with increasing prevalence [1]. Thus, health care expenditures are substantial; and considering our ageing population, they will continue to rise. HF morbidity and mortality are still high despite remarkable advancements in prevention and therapy [2]. Moreover, quality of life remains poor for HF patients [3] as HF causes injury and dysfunction of target organs, including the lung [4-7]. Although this affects primary disease management and outcome, the mechanisms underlying target organ injury in HF remain incompletely understood and hence, safe and efficient treatment strategies are limited. Regarding HF-associated lung complications, progress has been made in understanding the pathophysiology of pulmonary oedema, but other pulmonary complications of HF continue to challenge patients and clinicians alike.

Similar to several chronic lung diseases [8], elevated biomarker levels of inflammation are features of chronic HF. An augmentation in pro-inflammatory cytokines, including tumour necrosis factor alpha (TNF- $\alpha$ ) [9], has been demonstrated to play a role during HF progression, suggesting an involvement of inflammation during HF-mediated target organ damage [10]. We previously showed that therapeutically scavenging TNF- $\alpha$ using Etanercept attenuates target organ dysfunction in a mouse model of HF [7]. Therapeutic interventions aimed at limiting TNF- $\alpha$-mediated inflammation in chronic HF or lung diseases have yielded controversial results [11]. Considering this, we invested in understanding the molecular mechanism by which TNF- $\alpha$ signalling promotes target organ function during experimental HF [12]. Particularly, we showed that elevated TNF- $\alpha$ levels lead to considerable downregulation of the cystic fibrosis transmembrane regulator (CFTR) in the murine vasculature, heart, brain, and lung tissue [5]. The importance of proper CFTR function is appreciated in cystic fibrosis (CF) and chronic obstructive pulmonary disease (COPD). Here, CFTR protein dysfunction is common in the airways of affected patients [13]. In contrast to the genetic origin in CF, CFTR dysfunction in COPD is acquired since neutrophil elastase can induce alterations of CFTR expression, which 
correlate with disease severity [14]. Besides epithelial [15] and smooth muscle cells [5, 6], CFTR expression has been documented in several immune cells $[16,17]$. Peripheral blood monocytes isolated from patients heterozygous for the F508del CFTR mutation showed enhanced interleukin (IL)-8 secretion after activation compared to non-CF controls [18]. The latter was corroborated in macrophages isolated from Cftrknockout mice [19], suggesting a hyperinflammatory phenotype. Interestingly, pharmacological CFTR inhibition in macrophages increased secretion of pro-inflammatory cytokines [17], suggesting that acquired CFTR dysfunction (e.g., induced by HF, smoking or neutrophil elastase [14, 20, 21]) may contribute to hyperinflammatory immune responses. Since dysregulation of inflammation represents a hallmark of multiorgan manifestations of many diseases, including HF, we tested the hypothesis that murine HF associates with pulmonary CFTR dysfunction and concurrent tissue inflammation, which is correctable by CFTR targeting therapy.

\section{Material and Methods}

Materials : All chemical reagents and solutions were purchased from Fisher Scientific (Göteborg, Sweden), Saveen \& Werner (Limhamn, Sweden) or Sigma-Aldrich (Stockholm, Sweden) unless otherwise stated. Primers for qPCR were purchased from Eurofins (Ebersberg, Germany).

Animals: This investigation conforms with the Guide for Care and Use of Laboratory Animals published by the European Union (Directive 2010/63/EU) and the ARRIVE 2.0 guidelines. All animal care and experimental protocols were approved by the institutional animal ethics committee at Lund University (Dnr.: 5.8.18-08003/2017; 5.8.18-04938/2021) and were conducted in accordance with European animal protection laws. Commercially available male wild-type mice (12-14 weeks old; C57BL/6N) were purchased from Taconic (Lyngby, Denmark). All mice were housed under a standard 12h:12h light-dark cycle and had access to standard chow and water ad libitum. In the clinic, research into sex differences showed that HF prevalence is about 1.5-2x higher in men above 55 years of age compared to women [22]. Moreover, women have a higher probability of survival [23]. Females are therefore more protected from HF than males. For this reason, male mice that generally show a stronger phenotype were used in this study.

To ensure blinding, experiments were performed after the animals and samples had received codes that did not reveal the identity of the treatment. HF animals were assigned to vehicle or treatment groups using block randomization. To obey the rules for animal welfare, experimental groups were designed to minimize stress and guarantee maximal information using the lowest group size possible when calculated with a type I error rate of $\alpha=0.05(5 \%)$ and power of $1-\beta>0.8(80 \%)$ based on earlier studies [5, 24].

Myocardial infarction (MI): HF in mice was induced by experimental MI generated by permanent surgical ligation of the left anterior descending (LAD) coronary artery [12]. Briefly, mice were anesthetized with isoflurane $(1.5-2 \%$ in air), intubated with a 22 -gauge angiocatheter, and ventilated with room air at a rate of $120 \mathrm{bpm}, 250 \mu \mathrm{l}$ tidal volume, and $3 \mathrm{~cm}$ positive end expiratory pressure. The thorax and pericardium were opened, and the LAD was permanently ligated with 7-0 silk suture (Ågnthos, Sweden). Sham control mice underwent the same procedure without LAD ligation. Mice received pain medication $(2 \mu \mathrm{l} / \mathrm{g}$ mouse buprenorphine $0.05 \mathrm{mg} / \mathrm{ml}$ ) for up to three days post-surgery. This model shows stable cardiac injury 6 weeks after MI [12]. CFTR corrector treatment was initiated 10 weeks after MI (Supplemental Fig. 1 ). For 2 weeks, mice received daily intraperitoneal (i.p.) injections of Lumacaftor (Lum; $3 \mathrm{mg} / \mathrm{kg}$ in DMSO diluted 1:10 with sterile polyethylene glycol (PEG) in deionized (DI) water (50:50)) or were instilled with 50 $\mu \mathrm{l}$ Lum $(18 \mathrm{mg} / \mathrm{ml}$ in DMSO diluted 1:10 in sterile PBS) 5 times during the treatment period (orotracheal; o.t.). Group sizes were as follows: $\mathrm{N}=8$ for sham, $\mathrm{N}=10$ for $\mathrm{HF}, \mathrm{N}=10$ for $\mathrm{HF}+\mathrm{Lum}, \mathrm{N}=6$ for HF + Lum i.p., $\mathrm{N}=8$ for $\mathrm{HF}+$ Lum o.t. Not all animals were used for histology experiments.

Cardiac function assessment: Cardiac function was assessed using magnetic resonance (MR) imaging on a 9.4 T MR horizontal MR scanner equipped with Bruker BioSpec AVIII electronics, a quadrature volume resonator coil (112/087) for transmission and a $20 \mathrm{~mm}$ linear surface loop coil for reception (Bruker, Ettlingen, Germany), operating with ParaVision 6.0.1. Mice were anaesthetized with isoflurane in room air with $10 \%$ oxygen and kept at a respiration of $70-100 \mathrm{bpm}$ and at $36-37^{\circ} \mathrm{C}$ body temperature (sequence details in supplement). Image-based determination of ejection fraction (EF), stroke volume, cardiac output, end 
diastolic volume, end systolic volume, and left ventricle mass was performed with Segment (Medviso, Lund, Sweden).

Fluorescence activated cell sorting: After trans-cardiac perfusion, lung-heart blocks were extracted, and a broncho-alveolar lavage was performed by instilling sterile PBS. The left lung was cut into pieces and enzymatically digested in a DNAse-Collagenase XI mix under continuous agitation. After centrifugation, red blood cells were lysed, and the cell pellets were reconstituted in $\mathrm{F}_{\mathrm{c}}$ block prior to antibody staining (Supplemental Table 1 ). Data acquisition was carried out on a BD LSR II cytometer using FacsDiva software Vision 8.0 (BD Biosciences). Data analysis was performed with FlowJo software (version 10, TreeStar Inc., USA). Cells were plotted on forward versus side scatter and single cells were gated on FSC-A versus FSC-H linearity. Pulmonary macrophages were identified as Live, CD45 ${ }^{+}, \mathrm{B} 220^{-}, \mathrm{CD}_{11 b^{+}}, \mathrm{F} 4 / 80^{+}$cells (gating strategy: Supplemental Figure 2 ). Non-alveolar macrophages were identified as Live, CD $45^{+}$, B220- CD11b $^{+}, \mathrm{F} 4 / 80^{+}$, SiglecF- cells while alveolar macrophages were identified as Live, CD $45^{+}, \mathrm{B}_{2} 20^{-}$,

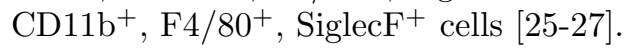

For CFTR staining, pulmonary cells were incubated with CFTR antibody and live/dead staining dye without reconstitution in $\mathrm{F}_{\mathrm{c}}$ block. After washing and centrifugation, cells were resuspended and incubated with a secondary goat anti-mouse AF488 antibody (Supplemental Table 1 ).

Hydroxyproline assay: Hydroxyproline content was measured using the "Hydroxyproline Assay Kit" as per manufacturer's instructions.

Cell culture: Murine macrophages (RAW246.7, ATCC TIB-71) were cultivated in high glucose DMEM supplemented with $10 \%$ heat inactivated fetal bovine serum and $1 \%$ Penicillin/Streptomycin. Cells were activated with $10 \mathrm{ng} / \mathrm{ml}$ phorbol 12-myristate 13-acetate (PMA, AdipoGen) for $48 \mathrm{~h}$ followed by a $24 \mathrm{~h}$ rest period before they were incubated with $10 \mu \mathrm{M}$ Lum (Cayman Chemicals) for $24 \mathrm{~h}$. In a second approach, Lum treatment was started at the same time as PMA-induced activation. Cells were harvested after $96 \mathrm{~h}$ and subjected to flow cytometry to determine CFTR surface expression.

Western Blotting, qPCR, and histological experiments: Standard procedures were utilized for experiments involving reverse transcription polymerase chain reaction (PCR), quantitative PCR, Western blotting, and histological experiments. See supplement for methodological details.

Data and Statistical Analysis: The data and statistical analysis comply with the recommendations on experimental design and analysis in pharmacology [28]. All data are expressed as mean \pm SEM, where $\mathrm{N}$ is the number of independent measures. Data were analysed using GraphPad Prism 8 software (San Diego, California). Data distribution was determined using Shapiro-Wilk test. For comparisons of 2 independent groups, a Student's t-test or Mann Whitney test was used. For comparison of multiple independent groups, one-way analysis of variance (ANOVA) or a Kruskal Wallis test was used, followed by a Tukey or Dunnettposthoc test. Differences were considered significant at $\mathrm{p}<0.05$.

\section{Results}

The pulmonary phenotype during HF is characterized by vascular remodelling and myeloid cell infiltration

Twelve weeks after MI, mice presented with cardiac dysfunction evidenced by significantly reduced EF (HF: $37.5 \% \pm 9.4 \%$ vs. sham: $63.8 \% \pm 5.9 \%$; Supplemental Table 3 ) and pulmonary structural alterations confined to the vasculature. HF mice exhibited markedly thicker blood vessel walls (Fig. 1a, b) and higher smooth muscle actin (SMA) mRNA (Supplemental Fig. 3 ) and protein levels (Fig. 1c ) compared to sham-operated controls. HF lungs did not differ macroscopically nor showed signs of fibrosis demonstrated by the lack of collagen accumulation assessed by Masson trichrome staining (Fig. 1d, e ) and hydroxyproline quantification (Fig. 1f ).

The apparent vascular remodelling was accompanied by higher monocyte/macrophage association with vascular structures in HF lungs as illustrated by monocyte/macrophage (MOMA) immunostaining in lung slices (Fig.2a, b ). Flow cytometric immune cell profiling of the HF lung revealed significantly higher cell numbers 
of $\mathrm{CD} 45^{\text {hi }}$ Ly6C $^{+}$SiglecF- cells (Fig. 2c, e) and CD $45^{\text {hi }}$ Ly6C $^{\text {hi }}$ SiglecF- cells in HF compared to Sham mice (Fig. 2d, e) , resembling infiltrating macrophages and pro-inflammatory monocytes. When analysing the activation profile of $\mathrm{F} 4 / 80^{+}$macrophages, we observed significantly higher cell numbers of classically-activated $\mathrm{CD} 80^{+}$macrophages in HF lungs (Fig. 2f, i ), indicative of a shift to a pro-inflammatory phenotype within the macrophage population. This increase was mainly driven by non-alveolar (SiglecF-) macrophages (Fig. $\mathbf{2 g}, \mathbf{i})$ as no difference was observed in the alveolar $\left(\operatorname{SiglecF}^{+}\right)$macrophage population (Fig. $\left.\mathbf{2 h}, \mathbf{i}\right)$.

Reduced pulmonary CFTR expression is a hallmark of the HF lung

The accumulation of non-alveolar classically-activated macrophages $\left(\mathrm{CD} 80^{+}\right.$SiglecF$\left.^{-}\right)$associated with markedly higher TNF- $\alpha$ protein levels in HF compared to sham lungs (Fig. 3a ). Since TNF- $\alpha$ potently reduces CFTR surface expression in different cell types [5, 29], we determined cell surface-specific CFTR expression in the lung by performing a flow cytometry-based CFTR staining approach with an antibody targeting membrane-associated, mature CFTR protein [30] (Supplemental Table 1 ). The overall number of surface$\mathrm{CFTR}^{+}$cells was significantly reduced in HF lungs (Fig. 3b, c ), which coincided with significantly lower expression levels of membrane-bound CFTR assessed by Western blotting (Fig. 3d ).

Pharmacological CFTR correction mitigates structural changes in the HF lung

We subjected a group of HF mice to CFTR corrector treatment using Lumacaftor (Lum), which acts as a chaperone improving CFTR protein folding and transport to the cell membrane and hence, increases cell surface CFTR protein expression [31, 32]. Systemic (i.p.) Lum administration 10 weeks post MI did not affect heart function (Supplemental Table 3 ), while significantly increasing the number of CFTR ${ }^{+}$cells in the HF lung (Fig. 4a, b ). Similarly, Western blot evaluation confirmed that the membrane-specific CFTR protein expression reached sham levels after two weeks of CFTR corrector treatment (Fig. 4c ). The overall number of $\mathrm{CFTR}^{+}$cells was not further enhanced by lung-specific, orotracheal (o.t.) Lum instillation (Fig. 4d ). However, o.t. treatment resulted in significant higher CFTR expression on the cell surface of CFTR ${ }^{+}$ lung cells as evidenced by increased median fluorescence intensity (MFI) in the o.t.-treated lungs compared to lungs from i.p.-treated HF mice (Fig. 4e, f ). Although not significant, we noted higher CFTR protein expression by Western blotting with o.t. application of Lum (Fig. $\mathbf{4 g}$ ).

CFTR correction attenuated alteration of the pulmonary vascular structure in the HF lung. Lum application mitigated the HF-associated thickening of pulmonary blood vessel walls (Fig 5a \& Supplemental Fig. 4 ) and led to significantly lower SMA protein levels (Fig. 5b ). This treatment effect was independent of application route supported by similar vessel wall thickness (Fig. 5c ) and SMA protein expression (Fig. 5d ) after both i.p. and o.t. Lum treatment. Immunofluorescent assessment of $\mathrm{MOMA}^{+}$cell distributions in lung slices verified an attenuation of the HF-associated elevation of monocytes/macrophages (Fig. 2a ) within and around the vessels after Lum treatment (Fig. 5e, f ).

Pharmacological CFTR correction promotes an anti-inflammatory phenotype of macrophages in the HF lung

Considering the high CFTR positivity of peripheral and pulmonary monocytes and macrophages (Supplemental Fig. 5A, 5B ), we explored the effects of pharmacological CFTR correction on macrophages in the lung. Both systemic and lung-specific Lum administration significantly increased the overall number of pulmonary macrophages (Supplemental Fig. 6A ) with larger effects after o.t. application. The treatment-associated increase of overall pulmonary macrophage counts was mainly mediated by increases of non-alveolar macrophages (Supplemental Fig. 6B ), which were more pronounced after o.t. application. In contrast to systemic administration, o.t. administered Lum markedly augmented the number of alveolar macrophages (Supplemental Fig. 6C ). To understand whether this increase in macrophages was beneficial or rather detrimental, we explored macrophage activation profiles by determining the proportion of classically- $\left(\mathrm{CD} 80^{+}\right)$and alternatively- $\left(\mathrm{CD} 206^{+}\right)$activated cells within the pulmonary macrophage population. The HF-associated augmentation of classically-activated macrophages was alleviated by therapeutic Lum administration irrespective of application route (Fig. 6a, d ). Likewise, therapeutic CFTR correction significantly attenuated the HF-associated increase of non-alveolar CD80 ${ }^{+}$ macrophages (Fig. 6b, e ). Interestingly, o.t. treated HF lungs presented with markedly higher proportions 
of $\mathrm{CD} 80^{+}$alveolar macrophages (Fig. 6c, f ), suggesting an application-induced pro-inflammatory response. In contrast to $\mathrm{CD} 80^{+}$macrophages, Lum induced higher proportions of alternatively-activated macrophages overall as well as alveolar and non-alveolar irrespective of treatment route (Fig. 6, Supplemental Fig. 7 andSupplemental Table 4 ). This is corroborated by increased pulmonary IL-10 mRNA expression after systemic Lum administration (Supplemental Fig. 8 ). In vitro, murine macrophages (RAW246.7 cells) presented with reduced CFTR positivity after PMA-induced activation, which was attenuated by CFTR correction with Lum (Supplemental Fig. 9 ), suggesting an interplay between CFTR surface expression and macrophage activation.

\section{Discussion}

This study describes an apparent lung phenotype during experimental HF characterized by vascular remodelling and tissue inflammation. For the first time, we show that pharmacological correction of CFTR mitigates the HF-induced downregulation of pulmonary CFTR expression and increases the proportion of $\mathrm{CFTR}^{+}$cells in the lung, normalises vessel wall thickness, and diminishes the HF-associated elevation of classically-activated non-alveolar macrophages within the lungs. Our data suggest pharmacological CFTR correction as promising approach to alleviate HF-induced inflammation in the lung.

The manifestation of HF in the lung is well-established. However, difficulties in the treatment of HF patients with chronic lung phenotypes remain, as standard therapies are often complicated by contraindications. Here, we verify a HF-mediated CFTR downregulation in the lung [5], a concept that may provide new mechanismbased treatment options for HF patients with pulmonary complications. Given the increasing evidence for an acquired CFTR dysfunction not only during HF but also in classic chronic lung diseases such as COPD and asthma [33], the indication that CFTR modulators may be useful therapeutics in the treatment of acquired CFTR abnormalities is certainly of interest to the field. First trials verified efficacy of the CFTR potentiator ivacaftor in COPD patients with chronic bronchitis [34]. Here, we describe beneficial effects of CFTR correction with Lum on lung inflammation and associated structural alterations during experimental HF. Specifically, Lum therapy attenuated the HF-associated increase in small vessel wall thickness, indicating beneficial effects on pulmonary arteriopathy, which often accompanies HF in patients with chronic left ventricular dysfunction [35], generally associating with increased risk of pulmonary complications and hence, overall poor disease outcome. Despite thickened pulmonary vessel walls, we did not observe higher collagen accumulation within HF lungs or around the pulmonary vasculature. In our experiments, we aim at obtaining physiological values for animal ventilation during surgery to avoid ventilator-induced lung injury [36], which cannot be excluded from other studies that reported additional structural alterations and higher collagen content in HF lungs in mice with comparable EF [37, 38].

Inflammation is a key player in both chronic heart and lung diseases and critically contributes to vasculopathies. Here, we find increased numbers of pro-inflammatory monocytes/macrophages infiltrating the HF lung and an accumulation of monocytes/macrophages around the pulmonary vasculature, suggesting inflammation-associated vascular remodelling. Monocytes/macrophages have been shown to be among the primary effectors of inflammation in pulmonary lesions, and lung interstitial macrophages play a major role in lung inflammation and dysfunction in several diseases. Monocytes expressing certain chemokine receptors have been shown to differentiate into interstitial perivascular macrophages, which secrete pro-inflammatory cytokines and contribute to vascular remodelling [39]. Whether changes in CFTR surface expression on circulating monocytes/macrophages mediates similar effects is an interesting question especially, considering their relatively high CFTR positivity compared to other immune cells, reported increased secretion of proinflammatory cytokines after pharmacological CFTR inhibition in macrophages [17], and the herein observed activation-induced CFTR surface reduction on macrophages.

HF leads to systemic TNF- $\alpha$ elevation in mice and men $[5,24,40]$, which negatively affects target organs, including the lung [40]. We previously showed that TNF- $\alpha$ sequestration with Etanercept attenuated the HF-associated reduction of pulmonary CFTR protein expression [5]. TNF- $\alpha$ was shown to mediate reduction of CFTR expression on the surface of different cell types [5, 29], suggesting that the herein detected HFassociated augmentation of pulmonary TNF- $\alpha$ might be directly linked to the observed overall CFTR down- 
regulation in the HF lung. TNF- $\alpha$, amongst other pro-inflammatory cytokines, induces M1-like macrophage phenotypes [41] and is secreted by classically-polarized CD80 ${ }^{+}$macrophages [42], which accumulate in the HF lung in our model. TNF- $\alpha$ sequestration using Etanercept was shown to reduce M1-type markers supported by decreases of CD40 and CD80 surface markers and increased expression of M2-type markers in human monocyte-derived macrophages [43]. Here, we find a similar lowering of $\mathrm{CD} 80^{+}$non-alveolar macrophages in the HF lung after Lum therapy, suggesting an intimate link between CFTR signalling and inflammation. Although direct Lum application to the lung resulted in higher CFTR expression on pulmonary CFTR ${ }^{+}$ cells, supporting higher corrector efficacy, increased CD $80^{+}$alveolar macrophage numbers that were observed with this treatment regimen may limit long-term benefits of lung-specific Lum application. CFTR correctorinduced increases of IL-10 in combination with the elevation of CD206 ${ }^{+}$cells in our model are suggestive of an involvement of CFTR in macrophage phenotype switching that promote a more restorative environment [42]. An alternative activation of human monocytes from CF patients after CFTR correction as evidenced by increased IL-10 secretion [44] corroborate our findings. Since CFTR alterations in pulmonary macrophages and monocyte-derived macrophages present with an exaggerated cytokine response to bacterial lipopolysaccharide [19] altered bactericidal activity [45], and adhesion [46], a direct role of CFTR in lung inflammation during HF is likely.

\section{Summary and Conclusion}

HF presents with an apparent lung phenotype characterized by thickened walls of small vessels within the lung and an elevation of classically-activated non-alveolar macrophages. Pharmacological CFTR correction with Lum attenuates HF-associated vascular alterations and lowers pro-inflammatory macrophage numbers, while promoting an alternatively-activated phenotype. Collectively, these data suggest pharmacological CFTR correction as promising approach to mitigate HF-induced pulmonary inflammation and associated structural alterations.

\section{References:}

1. Bueno H, Moura B, Lancellotti P, et al. The year in cardiovascular medicine 2020: heart failure and cardiomyopathies. Eur Heart J 2021; 42(6): p. 657-670.

2. Savarese G, Lund LH. Global Public Health Burden of Heart Failure. Card Fail Rev 2017; 3(1): p. 7-11.

3. Moradi M, Daneshi F, Behzadmehr R, et al. Quality of life of chronic heart failure patients: a systematic review and meta-analysis. Heart Fail Rev 2020; 25(6): p. 993-1006.

4. Harjola VP, Mullens W, Banaszewski M, et al. Organ dysfunction, injury and failure in acute heart failure: from pathophysiology to diagnosis and management. A review on behalf of the Acute Heart Failure Committee of the Heart Failure Association (HFA) of the European Society of Cardiology (ESC). Eur J Heart Fail 2017; 19(7): p. 821-836.

5. Meissner A, Yang J, Kroetsch JT, et al. Tumor necrosis factor-alpha-mediated downregulation of the cystic fibrosis transmembrane conductance regulator drives pathological sphingosine-1-phosphate signaling in a mouse model of heart failure. Circulation 2012; 125(22): p. 2739-50.

6. Lidington D, Fares JC, Uhl FE, et al. CFTR Therapeutics Normalize Cerebral Perfusion Deficits in Mouse Models of Heart Failure and Subarachnoid Hemorrhage. JACC Basic Transl Sci 2019; 4(8): p. 940-958.

7. Meissner A, Visanji NP, Momen MA, et al. Tumor Necrosis Factor-alpha Underlies Loss of Cortical Dendritic Spine Density in a Mouse Model of Congestive Heart Failure. J Am Heart Assoc 2015; 4(5).

8. Chung KF. Cytokines in chronic obstructive pulmonary disease. Eur Respir J Suppl 2001; 34: p. 50s-59s.

9. Mann DL. Inflammatory mediators and the failing heart: past, present, and the foreseeable future. Circ Res 2002; 91(11): p. 988-98.

10. Mann DL. Innate immunity and the failing heart: the cytokine hypothesis revisited. Circ Res 2015; 116(7): p. 1254-68. 
11. Mann DL, McMurray JJ, Packer M, et al. Targeted anticytokine therapy in patients with chronic heart failure: results of the Randomized Etanercept Worldwide Evaluation (RENEWAL). Circulation 2004; 109(13): p. 1594-602.

12. Yang J, Noyan-Ashraf MH, Meissner A, et al. Proximal cerebral arteries develop myogenic responsiveness in heart failure via tumor necrosis factor-alpha-dependent activation of sphingosine-1-phosphate signaling. Circulation 2012; 126(2): p. 196-206.

13. Dransfield MT, Wilhelm AM, Flanagan B, et al. Acquired cystic fibrosis transmembrane conductance regulator dysfunction in the lower airways in COPD. Chest 2013; 144(2): p. 498-506.

14. Rab A, Rowe SM, Raju SV, et al. Cigarette smoke and CFTR: implications in the pathogenesis of COPD. Am J Physiol Lung Cell Mol Physiol 2013; 305(8): p. L530-41.

15. Engelhardt JF, Zepeda M, Cohn JA, et al. Expression of the cystic fibrosis gene in adult human lung. J Clin Invest 1994; 93(2): p. 737-49.

16. Yoshimura K, Nakamura H, Trapnell BC, et al. Expression of the cystic fibrosis transmembrane conductance regulator gene in cells of non-epithelial origin. Nucleic Acids Res 1991; 19(19): p. 5417-23.

17. Di A, Brown ME, Deriy LV, et al. CFTR regulates phagosome acidification in macrophages and alters bactericidal activity. Nat Cell Biol 2006; 8(9): p. 933-44.

18. Zaman MM, Gelrud A, Junaidi O, et al. Interleukin 8 secretion from monocytes of subjects heterozygous for the deltaF508 cystic fibrosis transmembrane conductance regulator gene mutation is altered. Clin Diagn Lab Immunol 2004; 11(5): p. 819-24.

19. Bruscia EM, Zhang PX, Ferreira E, et al. Macrophages directly contribute to the exaggerated inflammatory response in cystic fibrosis transmembrane conductance regulator-/- mice. Am J Respir Cell Mol Biol 2009; 40(3): p. 295-304.

20. Raju SV, Jackson PL, Courville CA, et al. Cigarette smoke induces systemic defects in cystic fibrosis transmembrane conductance regulator function. Am J Respir Crit Care Med 2013; 188(11): p. 1321-30.

21. Marklew AJ, Patel W, Moore PJ, et al. Cigarette Smoke Exposure Induces Retrograde Trafficking of CFTR to the Endoplasmic Reticulum. Sci Rep 2019; 9(1): p. 13655.

22. Tadic M, Cuspidi C, Plein S, et al. Sex and Heart Failure with Preserved Ejection Fraction: From Pathophysiology to Clinical Studies. J Clin Med 2019; 8(6).

23. Savarese G, D'Amario D. Sex Differences in Heart Failure. Adv Exp Med Biol 2018; 1065: p. 529-544.

24. Yagi K, Lidington D, Wan H, et al. Therapeutically Targeting Tumor Necrosis Factor-alpha/Sphingosine1-Phosphate Signaling Corrects Myogenic Reactivity in Subarachnoid Hemorrhage. Stroke 2015; 46(8): p. 2260-70.

25. Hu L, Chen Z, Li L, et al. Resveratrol decreases CD45(+) CD206(-) subtype macrophages in LPS-induced murine acute lung injury by SOCS3 signalling pathway. J Cell Mol Med 2019; 23(12): p. 8101-8113.

26. Huaux F, De Gussem V, Lebrun A, et al. New interplay between interstitial and alveolar macrophages explains pulmonary alveolar proteinosis (PAP) induced by indium tin oxide particles. Arch Toxicol 2018; 92(4): p. 1349-1361.

27. Misharin AV, Morales-Nebreda L, Mutlu GM, et al. Flow cytometric analysis of macrophages and dendritic cell subsets in the mouse lung. Am J Respir Cell Mol Biol 2013; 49(4): p. 503-10.

28. Curtis MJ, Alexander S, Cirino G, et al. Experimental design and analysis and their reporting II: updated and simplified guidance for authors and peer reviewers. Br J Pharmacol 2018; 175(7): p. 987-993. 
29. Malik FA, Meissner A, Semenkov I, et al. Sphingosine-1-Phosphate Is a Novel Regulator of Cystic Fibrosis Transmembrane Conductance Regulator (CFTR) Activity. PLoS One 2015; 10(6): p. e0130313.

30. Csanady L, Vergani P, Gadsby DC. Structure, Gating, and Regulation of the Cftr Anion Channel. Physiol Rev 2019; 99(1): p. 707-738.

31. Gentzsch M, Mall MA. Ion Channel Modulators in Cystic Fibrosis. Chest 2018; 154(2): p. 383-393.

32. Van Goor F, Hadida S, Grootenhuis PD, et al. Correction of the F508del-CFTR protein processing defect in vitro by the investigational drug VX-809. Proc Natl Acad Sci U S A 2011; 108(46): p. 18843-8.

33. Solomon GM, Fu L, Rowe SM, et al. The therapeutic potential of CFTR modulators for COPD and other airway diseases. Curr Opin Pharmacol 2017; 34: p. 132-139.

34. Solomon GM, Hathorne H, Liu B, et al. Pilot evaluation of ivacaftor for chronic bronchitis. Lancet Respir Med 2016; 4(6): p. e32-3.

35. Gerges M, Gerges C, Pistritto AM, et al. Pulmonary Hypertension in Heart Failure. Epidemiology, Right Ventricular Function, and Survival. Am J Respir Crit Care Med 2015; 192(10): p. 1234-46.

36. Hamlington KL, Smith BJ, Dunn CM, et al. Linking lung function to structural damage of alveolar epithelium in ventilator-induced lung injury. Respir Physiol Neurobiol 2018; 255: p. 22-29.

37. Dayeh NR, Tardif JC, Shi Y, et al. Echocardiographic validation of pulmonary hypertension due to heart failure with reduced ejection fraction in mice. Sci Rep 2018; 8(1): p. 1363.

38. Jasmin JF, Mercier I, Hnasko R, et al. Lung remodeling and pulmonary hypertension after myocardial infarction: pathogenic role of reduced caveolin expression. Cardiovasc Res 2004; 63(4): p. 747-55.

39. Florentin J, Coppin E, Vasamsetti SB, et al. Inflammatory Macrophage Expansion in Pulmonary Hypertension Depends upon Mobilization of Blood-Borne Monocytes. J Immunol 2018; 200(10): p. 36123625 .

40. Schumacher SM, Naga Prasad SV. Tumor Necrosis Factor-alpha in Heart Failure: an Updated Review. Curr Cardiol Rep 2018; 20(11): p. 117.

41. Arora S, Dev K, Agarwal B, et al. Macrophages: Their role, activation and polarization in pulmonary diseases. Immunobiology 2018; 223(4-5): p. 383-396.

42. Shapouri-Moghaddam A, Mohammadian S, Vazini H, et al. Macrophage plasticity, polarization, and function in health and disease. J Cell Physiol 2018; 233(9): p. 6425-6440.

43. Degboe Y, Rauwel B, Baron M, et al. Polarization of Rheumatoid Macrophages by TNF Targeting Through an IL-10/STAT3 Mechanism. Front Immunol 2019; 10: p. 3.

44. Jarosz-Griffiths HH, Scambler T, Wong CH, et al. Different CFTR modulator combinations downregulate inflammation differently in cystic fibrosis. Elife 2020; 9.

45. Kopp BT, Abdulrahman BA, Khweek AA, et al. Exaggerated inflammatory responses mediated by Burkholderia cenocepacia in human macrophages derived from Cystic fibrosis patients. Biochem Biophys Res Commun 2012; 424(2): p. 221-7.

46. Sorio C, Montresor A, Bolomini-Vittori M, et al. Mutations of Cystic Fibrosis Transmembrane Conductance Regulator Gene Cause a Monocyte-Selective Adhesion Deficiency. Am J Respir Crit Care Med 2016; 193(10): p. 1123-33.

Figure Legends

Fig. 1: Heart failure-associated structural changes in the lung are confined to blood vessels. a) Haematoxylin and Eosin staining of lungs from sham and heart failure (HF) mice and b)quantification of the vessel wall thickness of small vessels. Arrows indicate vessel walls. scale bar $20 \mu \mathrm{m}$. c) Representative 
Western Blot and quantification of the smooth muscle actin (SMA) protein expression in lung tissue from sham and HF mice. d) Masson Trichrome staining of lungs from sham and HF mice and e) qualitative quantification of the amount of collagen staining. Arrows indicate collagen, stained in blue. scale bar $20 \mu \mathrm{m}$. f) Quantification of the hydroxyproline content of lung tissue from sham and HF mice. Data expressed as mean \pm SEM. ${ }^{*}$ denotes p [?] 0.05 for single, unpaired comparisons.

Fig. 2: Heart failure associates to lung infiltration of $\mathrm{CD}^{+} 0^{+}$pro-inflammatory macrophages. a) Representative images of lung sections from sham and heart failure (HF) mice that were stained for monocyte/macrophages (MOMA) in red, smooth muscle actin (SMA) in green, DAPI stained nuclei in blue, scale bar $20 \mu \mathrm{m}$. b) Quantification of the percentage of MOMA positive cells.c) Flow cytometry results representing the number of $\mathrm{CD}_{4} 5^{\mathrm{hi}}, \mathrm{Ly}_{6 \mathrm{C}^{+}}$SiglecF- and d) $\mathrm{CD}^{-} 5^{\mathrm{hi}}$, Ly6Chi ${ }^{\mathrm{higlecF}}{ }^{-}$macrophages ande) representative dot blots of the Ly6C and SiglecF expression of $\mathrm{F} 4 / 80^{+}$macrophages in the lung of sham and HF mice.f) $\mathrm{F} 4 / 80^{+}$, CD80 ${ }^{+}$classically activated macrophages, g) F4 $/ 80^{+}$, CD $80^{+}$, SiglecF-classicallyactivated non-alveolar macrophages, h)F $4 / 80^{+}, \mathrm{CD} 80^{+}$, Siglec $\mathrm{F}^{+}$classically-activated alveolar macrophages in the lung of sham and HF mice and $\mathbf{i}$ ) representative dot blots of the SiglecF and CD80 expression of F4/80 ${ }^{+}$ macrophages in the lung of sham and HF mice. Data expressed as mean \pm SEM. * denotes $\mathrm{p}[?] \quad 0.05$ for single, unpaired comparisons.

Fig. 3: Pulmonary tumour necrosis factor alpha increase is accompanied by decreased cystic fibrosis transmembrane regulator expression in the heart failure lung. a) Representative Western Blot and b) quantification of tumour necrosis factor alpha (TNF- $\alpha$ ) expression in the lungs of sham and heart failure (HF) mice. c) Percentage of cystic fibrosis transmembrane regulator (CFTR) positive cells in the lungs of sham and HF mice and c) representative dot plots. d) Representative Western Blot and quantification of CFTR protein expression in the lungs of sham and HF mice. Data expressed as mean \pm SEM. * denotes p [?] 0.05 for single, unpaired comparisons.

Fig. 4: Systemic application of cystic fibrosis transmembrane regulator (CFTR) correctors increases pulmonary CFTR expression. a)Percentage of $\mathrm{CFTR}^{+}$cells in the lungs of sham, heart failure (HF), and Lumacaftor (Lum) treated (intraperitoneally (i.p.)) HF mice and b) representative dot plots. c)Representative Western Blot and d) quantification of the CFTR expression in the lungs of sham, HF, and Lumacaftor treated (i.p.) HF mice. e) Percentage of $\mathrm{CFTR}^{+}$cells in the lungs of HF mice treated with Lumacaftor either i.p. or orotracheally (o.t.), median fluorescence intensity and f) representative histograms of $\mathrm{CFTR}^{+}$cells in the lungs of HF mice treated with Lumacaftor either i.p. or o.t. g) Representative Western Blot and quantification of the CFTR expression in the lungs of HF mice treated with Lumacaftor either i.p. or o.t. Data expressed as mean +- SEM. In (a) and (c), * denotes p [?] 0.05 relative to sham, $\$$ denotes p [?] 0.05 relative to HF after one-way ANOVA followed by Tukey's post-hoc testing; in (e), $\$$ denotes $\mathrm{p}$ [?] 0.05 for single, unpaired comparisons.

Fig. 5: Cystic fibrosis transmembrane regulator correction mitigates heart failure-associated alteration of pulmonary vascular structure. a) Quantification of the vessel wall thickness of smaller vessels in the lungs of sham, heart failure (HF), and Lumacaftor (Lum) treated HF mice. b)Representative Western Blot and quantification of the smooth muscle actin (SMA) expression in lung tissue from sham, HF, and Lum treated HF mice. c) Quantification of the vessel wall thickness of smaller vessels in the lungs of Lumacaftor (Lum) treated (intraperitoneally (i.p.) or orotracheally (o.t.)) HF mice. d) Representative Western Blot and quantification of SMA expression in lung tissue from Lum treated (i.p. and o.t.) HF mice. The dotted line in indicates the level of HF mice. e) Representative images of lung sections from Lum treated (i.p. or o.t.) HF mice that were stained for monocyte/macrophages (MOMA, red) and SMA (green) and f) its quantification. DAPI stained nuclei in blue. The dotted line in f) indicates the level of HF mice. MOMA positive cells within the vessels indicated by white arrows. Scale bar $20 \mu \mathrm{m}$. Data expressed as mean \pm SEM. ${ }^{*}$ denotes p [?] 0.05 relative to sham, $\$$ denotes $\mathrm{p}$ [?] 0.05 relative to HF after one-way ANOVA followed by Tukey's post-hoc testing.

Fig. 6: Cystic fibrosis transmembrane regulator correction normalizes levels of non-alveolar macrophages and increases CD206 ${ }^{+}$alveolar macrophages. a, d) Percentage of CD $80^{+}$and CD206 ${ }^{+}$ 
pulmonary $\mathrm{F} 4 / 80^{+}$-macrophages of sham, heart failure (HF), and Lumacaftor (Lum) treated ((intraperitoneally (i.p.) or orotracheally (o.t.)) HF mice. b, e) Percentage of $\mathrm{CD} 80^{+}$and $\mathrm{CD} 206^{+}$pulmonary non-alveolar $\mathrm{F} 4 / 80^{+}$and SiglecF $\mathrm{F}^{-}$macrophages of sham, HF, and Lum treated (i.p. and o.t.) HF mice. c, f)Percentage of $\mathrm{CD} 80^{+}$and $\mathrm{CD} 206^{+}$pulmonary alveolar $\mathrm{F} 4 / 80^{+}$and $\mathrm{SiglecF}^{+}$macrophages of sham, HF, and Lum treated (i.p. and o.t.) HF mice. Data expressed as mean +- SEM. In (a, d), * denotes p [?] 0.05 relative to sham, $\$$ denotes $\mathrm{P}[?] 0.05$ relative to HF after one-way ANOVA followed by Tukey's post-hoc testing; in (b, c, e) and (f), ${ }^{*}$ denotes p [?] 0.05 relative to sham, $\$$ denotes P [?] 0.05 relative to HF after Kruskal Wallis followed by Dunnett's post-hoc testing.

a)

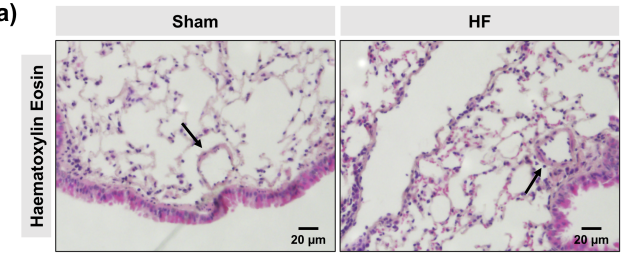

d)

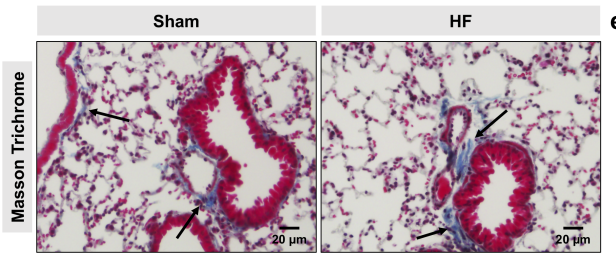

b)

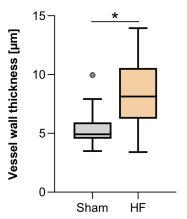

e)

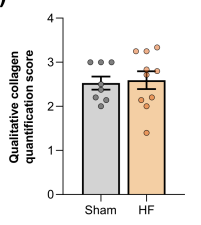

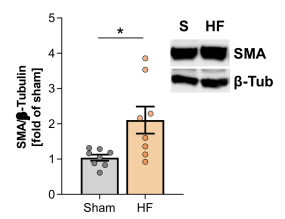

f)

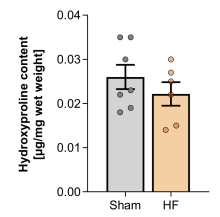


a)

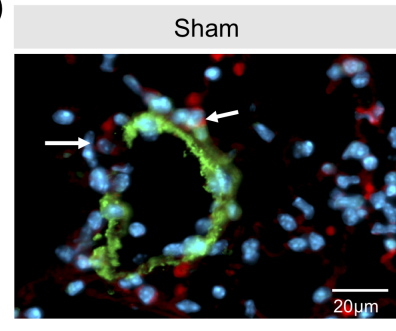

HF

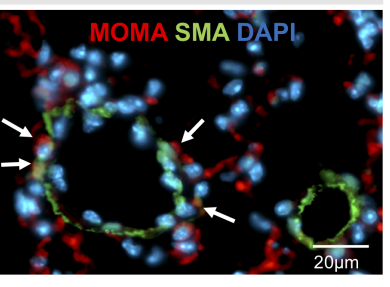

b)

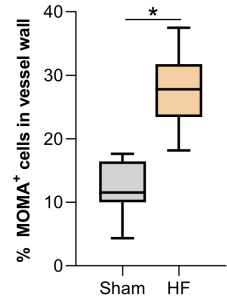

c)

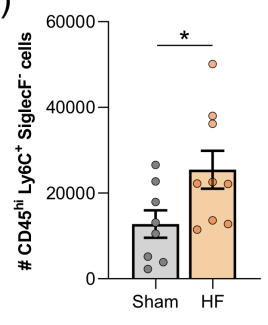

d)

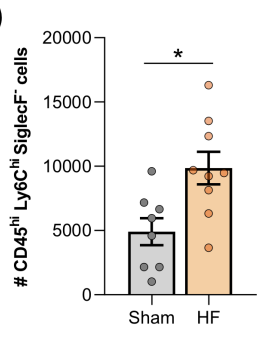

e)

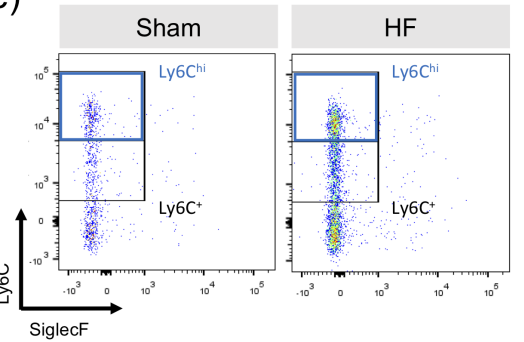

i)
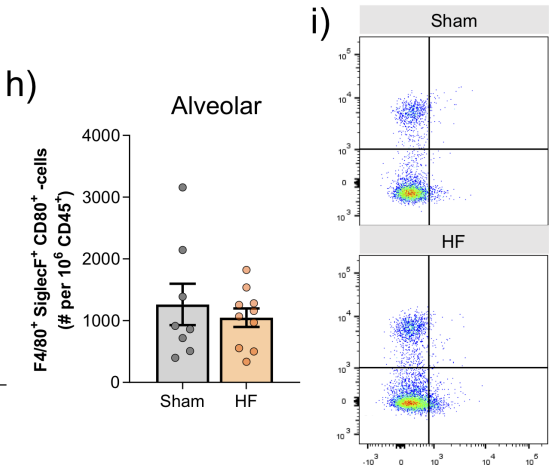

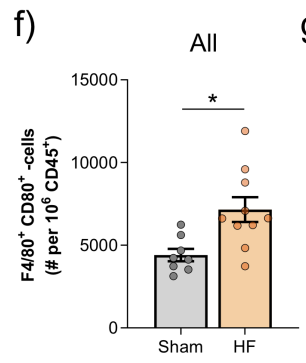

g)

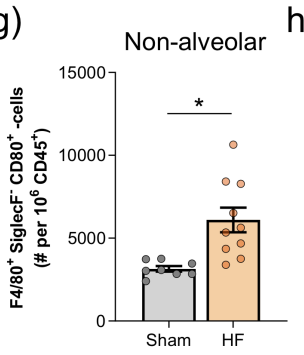

b)

a)
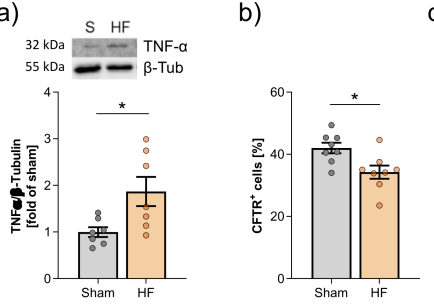

c)

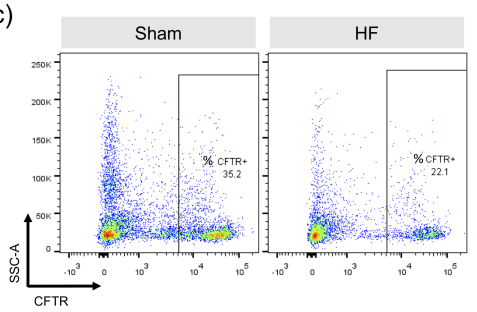

d) 


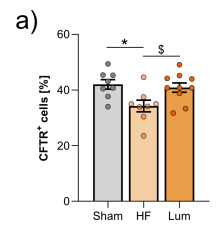

b)

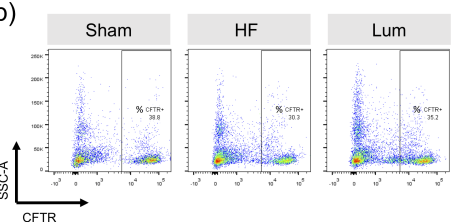

d)

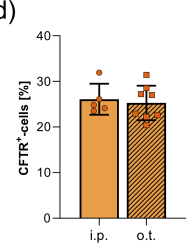

e)

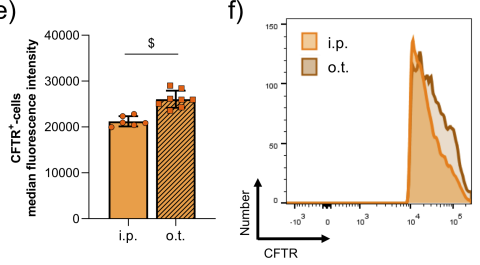

c)

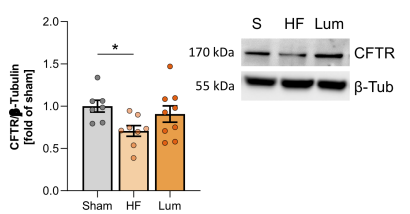

g)

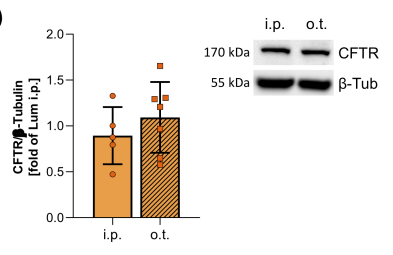

a)

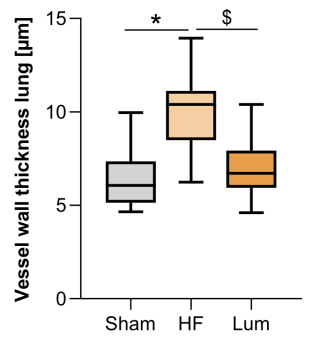

c)

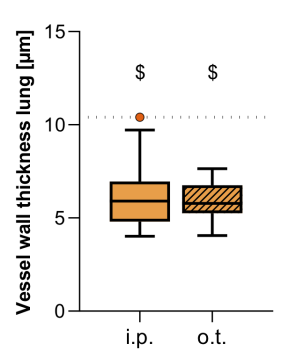

b)

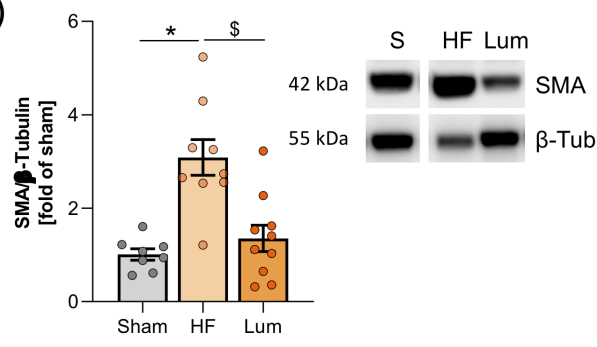

d)

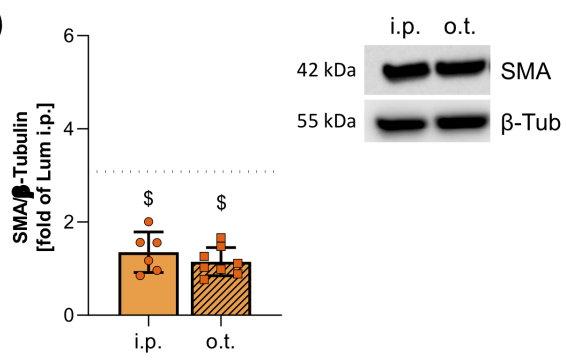

e)

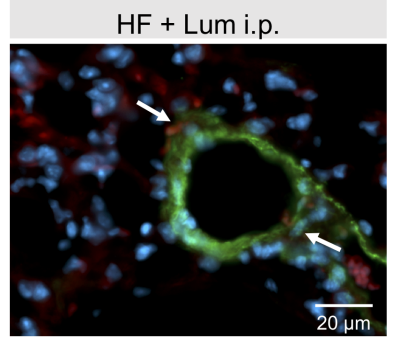

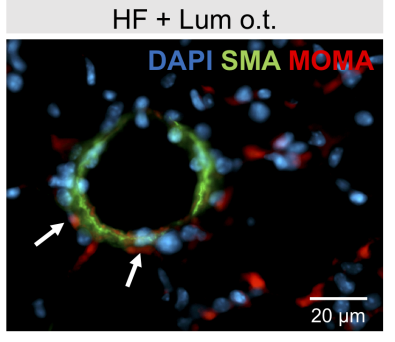

f)

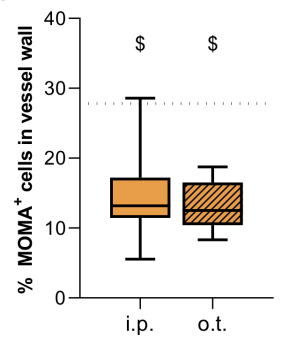


a)

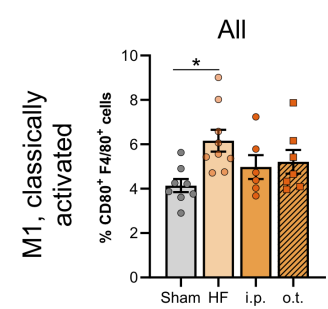

d)

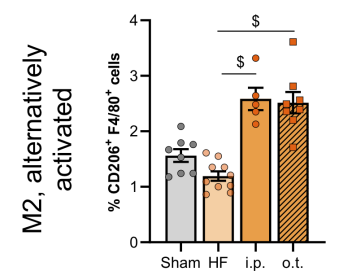

b)

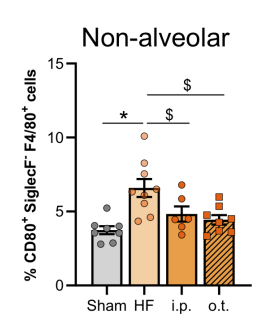

e)

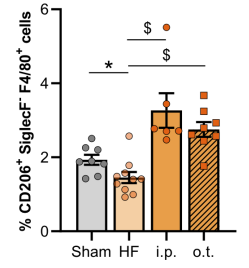

c)

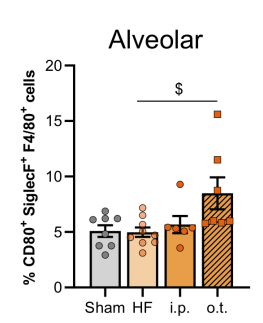

f)

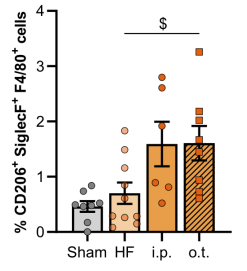

